\title{
EUROPEAN PAEDIATRIC SURGEONS' ASSOCIATION SURVEY ON THE MANAGEMENT OF HIRSCHSPRUNG'S DISEASE
}

Augusto Zani ${ }^{1}$, Simon Eaton ${ }^{2}$, Francesco Morini ${ }^{3}$, Prem Puri ${ }^{4}$, Risto Rintala ${ }^{5}$, Ernest van Heurn ${ }^{6}$, Marija Lukac ${ }^{7}$, Pietro Bagolan ${ }^{3}$, Joachim Kuebler ${ }^{8}$, Florian Friedmacher ${ }^{9}$, Rene Wijnen ${ }^{10}$, Juan Tovar $^{11}$, Michael Hoellwarth ${ }^{9}$, Agostino Pierro ${ }^{1}$, on behalf of the EUPSA Network Office

${ }^{1}$ Division of General and Thoracic Surgery, The Hospital for Sick Children, Toronto, ON, Canada

${ }^{2}$ Department of Pediatric Surgery, UCL - Institute of Child Health, London, UK

${ }^{3}$ Department of Medical and Surgical Neonatology, Bambino Gesù Children's Hospital, Rome, Italy

${ }^{4}$ Department of Paediatric Surgery, National Children's Research Centre, Dublin, Ireland ${ }^{5}$ Department of Paediatric Surgery, Hospital for Children and Adolescents, Helsinki, Finland

${ }^{6}$ Paediatric Surgical Center of Amsterdam, Emma Children's Hospital AMC \& VU University Medical Center, Amsterdam, The Netherlands

${ }^{7}$ Department of Pediatric Surgery, Faculty of Medicine, Belgrade, Serbia

${ }^{8}$ Department of Pediatric Surgery, Hannover Medical School, Hannover, Niedersachsen, Germany

${ }^{9}$ Department of Paediatric and Adolescent Surgery, Medical University of Graz, Graz, Austria

${ }^{10}$ Department of Pediatric Surgery, Sophia Children's Hospital, ErasmusMC, Rotterdam, The Netherlands

${ }^{11}$ Department of Pediatric Surgery, Hospital Universitario La Paz, Madrid, Spain 


\section{Abstract}

Aim This study aims to define patterns of Hirschsprung disease (HD) management.

Methods An online questionnaire was sent to all European Paediatric Surgeons' Association (EUPSA) members.

Results A total of 294 members (61 countries) answered (response rate: 61\%). Diagnosis: All respondents perform rectal biopsies (61\% rectal suction [RSBs], 39\% open full-thickness), $96 \%$ contrast enema, and 31\% anorectal manometry. At RSB, 17\% take the most distal biopsy $1 \mathrm{~cm}$ above the dentate line, $34 \%$ take $2 \mathrm{~cm}, 30 \%$ take $3 \mathrm{~cm}$, and $19 \%$ take $>3 \mathrm{~cm}$. Rectal biopsy staining's are hematoxylin/eosin (77\%), acetylcholinesterase (74\%), calretinin (31\%), S100 (2\%), nicotinamide adenine dinucleotide- tetrazolium reductase (2\%), succinate dehydrogenase (1\%), and neuron-specific enolase (1\%). A total of $85 \%$ respondents recognize entities including hypoganglionosis (69\%), intestinal neuronal dysplasia (55\%), and ultrashort segment HD (50\%). Surgery: pull through (PT) is performed at diagnosis by $33 \%$ or delayed by $67 \%$ ( 4 months or $>5$ kg). Awaiting definitive surgery, $77 \%$ perform rectal irrigations, $22 \%$ rectal dilatation/ stimulations, and 33\% perform a stoma. The preferred type of PT is the Soave approach (65\%), performed with transanal technique by $70 \%$ respondents. If symptoms persist after PT, most opt for conservative approach (enemas/laxatives $=76 \%$; botulinum toxin $=27 \%$ ), $30 \%$ would redo the PT. Total colonic aganglionosis: PT is performed in neonates (4\%), at 1 to 6 months (29\%), 6 to 12 months (37\%) or older (30\%). If required, a stoma is sited in the ileum (31\%), according to intraoperative biopsies (54\%), macroscopic impression (13\%), and radiology (2\%). Duhamel PT is performed by $52 \%$, Soave by $31 \%$, and Swenson by $17 \%$. Overall, $31 \%$ would perform a J-pouch. 
Conclusions Most aspects of HD management lack consensus with wide variations in obtaining a diagnosis. Transanal Soave PT is the most common technique in standard segment HD. Guidelines should be developed to avoid such variability in management and to facilitate research studies.

\section{Introduction}

Hirschsprung disease (HD), also known as congenital mega-colon, is a disorder of the enteric nervous system character-ized by the absence of ganglion cells in the distal colon resulting in functional obstruction. The first description of this condition dates back to 1886, when Harald Hirsch-sprung, a Danish pediatrician, presented the first portrayal of congenital megacolon at the Society of Pediatrics in Berlin.1 Since then an abundant literature has grown around the subject, and given the many controversial aspects of HD management, several surveys of practice were reported by various groups.

The Surgical Section of the American Academy of Pediat-rics performed a survey of its members between 1975 and 1976 to accumulate their combined experience on HD.2 This was the first survey of practice reported in the literature: 33 surgeons or groups of surgeons returned completed forms on 1,196 patients and 181 pediatric surgeons returned individ-ual preference sheets. Almost 30 years later, the American Pediatric Surgical Association (APSA) sent a 12-question survey to their members investigating the contemporary practice patterns in the surgical management of HD.3 The main change noticed between the two American surveys were about surgery that had shifted from a multistage approach using the Swenson or Duhamel techniques, 2 to a one-stage minimally invasive approach using either the laparoscopic or transanal approaches.3 In the United King-dom, two surveys of practice were conducted in 
1998 and in 2010, respectively.4,5 The first survey highlighted wide var-iations in preferred management strategies that led to rec-ommendations for regional subspecialization with dedicated HD surgeons performing definitive surgery.4 Similar to the North American experience, several changes in management strategy were also noticed between the two United Kingdom surveys with a similar trend toward the use of laparoscopy but not of transanal pull-through (PT).5 In Japan, three surveys were performed between 1978 and 2002.6-8 The latest survey reflected the changes in practice following the introduction of both laparoscopic surgery and transanal endorectal PT and summarized the analysis of 3,852 HD patients over 30 years.

The above-mentioned surveys, as well as others published in the literature, were conducted to investigate the practice patterns of a specific condition among a group of surgeons and to monitor the changes in practice that occurred over time. The aim of the present study was to analyze and report for the first time the patterns of HD management among the members of the European Pediatric Surgeons' Association.

\section{Methods}

Following approval of the European Paediatric Surgeons' Association (EUPSA) Executive Board and the EUPSA Network Office, 507 members were contacted via email and asked to fill out a questionnaire on the management of HD using SurveyMonkey (SurveyMonkey, Palo Alto, California), an online survey platform. Response anonymity was guaranteed by the fact that survey creator and analyzer worked independently. The questionnaire focused on various 
aspects of HD, such as diagnosis and surgery, and of specific patients, such as those with trisomy 21 and total colonic aganglionosis. A total of 25 EUPSA members opted out either because retired or because practicing only pediatric urology or because they do not manage HD patients. Of the remaining 482 members, 294 (61\%) completed the questionnaire.

Respondents were invited to fill in their position (Head of Department/Permanent Staff or Consultant/Trainee) and country of practice. Of the 294 respondents, 3 delegates did not disclose their degree, whereas 86 were head of the department (29\%), 171 consultants (59\%), and 34 trainees (12\%). On 287 questionnaires, respondents reported their country of origin: 226 (79\%) were from 33 European countries and 61 (21\%) from 28 non-European countries.

\section{Results}

Centre: Overall, 129 (45\%) respondents work in centres that treat less than 10 cases of HD a year, $122(42 \%)$ reported working in a centre that treats 10 to $20 \mathrm{HD}$ cases a year, and 38 (13\%) in a centre that treats more than 20 HD cases a year.

Diagnosis: In the workup of patients with suspected HD, all respondents (100\%) perform rectal biopsies, $96 \%$ request a contrast enema, and 31\% do anorectal manometry. Rectal biopsies are ob-tained using the suction technique by $61 \%$ respondents and via open full-thickness by the other $39 \%$ respondents; the number of specimens that are routinely taken is 3 by 55 respondents, 2 by $28 \%$, more than 3 by $13 \%$, and 1 by $4 \%$ (Fig. $1 \mathrm{~A}$ ). A total of $34 \%$ of surgeons take the most distal biopsy at $2 \mathrm{~cm}$ from the dentate line, $30 \%$ at $3 \mathrm{~cm}, 19 \%$ at more than $3 \mathrm{~cm}$, and $17 \%$ at $1 \mathrm{~cm}$ ( Fig. 1B). The most used histological and immunohistochemical methods used for HD diagnosis are hematoxylin/eosin (77\%), acetylcholinester $\neg$ ase (74\%), and calretinin 
staining (31\%); other markers are less commonly used: S100 (2\%), nicotinamide adenine dinu-cleotide-tetrazolium reductase (2\%), succinate dehydroge-nase (1\%), neuron-specific enolase (1\%), and lactate dehydrogenase (1\%) ( Fig. 1C). To receive biopsy reports it takes less than 24 hours for $9 \%$ of surgeons, 24 to 48 hours for $30 \%, 3$ to 5 days for $35 \%$, and more than 5 days for $29 \%$ ( Fig. 1D). The majority of surgeons (85\%) recognize the existence of at least one controversial entity including hypo-ganglionosis (69\%), intestinal neuronal dysplasia (55\%), and ultrashort segment HD (50\%) Surgery: In a neonate with confirmed HD, the next step in manage-ment for the majority of respondents $(67 \%)$ is to perform a PT immediately. The other $33 \%$ of respondents instead delay the PT for a median of 3 months (interquartile range: $2-5$ ) or when the infant is more than $5 \mathrm{~kg}$. While waiting for surgery, the bowel is maintained decompressed with rectal irrigations by $77 \%$ respondents, with rectal dilatation/stimulations by $22 \%$, whereas $33 \%$ perform a stoma. If a stoma is created in a patient with typical rectosigmoid HD, $34 \%$ perform a leveling stoma, $30 \%$ site it in the sigmoid colon, $26 \%$ in the right transverse colon, and $10 \%$ in the ileum.

In the case of standard length aganglionic segment, the preferred type of PT is the Soave approach (65\%, Fig. 2), which is performed with the transanal technique by $70 \%$ respondents, with laparoscopy by $20 \%$, and by the open technique by $10 \%$. The Swenson PT is the approach selected by $19 \%$ respondents, is performed with the transanal tech $\neg$ nique by $58 \%$, laparoscopic by $35 \%$, and as an open technique by $7 \%$. The Duhamel PT is performed by $16 \%$ respondents, and is performed with the open technique by $53 \%$, with laparoscopy by $25 \%$, and as transanal by $22 \%$. 
The PT is planned to happen in the neonatal period by $20 \%$ respondents, between 1 and 6 months of age by $62 \%$, between 6 months and 1 year of age by $15 \%$, and older than a year by $3 \%$.

If symptoms persist after a "successful" PT, the majority of respondents opt for a conservative approach with enemas and laxatives (76\%) and/or botulinum toxin injection (27\%), whereas $30 \%$ would redo the PT. Most surgeons (93\%) do not change their surgical approach in patients with trisomy 21 . The surgeons that modify their approach in the case of a patient with trisomy 21 mostly delay the PT, create a colostomy from the beginning, and leave it for a longer period of time. Total colonic aganglionosis (TCA): In patients with an established diagnosis of total colonic aganglionosis (TCA), only $4 \%$ respondents perform the PT during the neonatal period, $29 \%$ between 1 and 6 months of age, 37\% between 6 months and 1 year of age, and 30\% when the patient is older than a year of age. If a stoma is required, it is sited always in the ileum by $31 \%$ respondents or according to the intraoperative biopsies by $54 \%$ or according to the macroscopic impression at surgery by $13 \%$, or based on the radiological findings by $2 \%$. The preferred PT in patients with TCA is the Duhamel procedure (52\%), followed by Soave (31\%) and Swenson (17\%). Overall, 31\% responders would perform a J-pouch in a patient with TCA.

\section{Discussion}

The results of this first survey of practice among members of the EUPSA show that most aspects of HD management lack consensus. The quality of data collected with this question $\neg$ naire derives from the seniority of the respondents ( $88 \%$ senior surgeons) and the internationality of their 61 centers (33 European and 28 non-European). Moreover, the majority of respondents 
(55\%) practice in centers that treat more than $10 \mathrm{HD}$ cases a year. The response rate obtained with the online platform was satisfactory (61\%) and higher than in other surveys on the same subject.2,3,5 The advantages of this method are that it reduces time and costs for data input, eliminates interviewer-related bias (more privacy for the respondent), minimizes errors during data transcription and controls answer validity. 9 On the other hand, we ac - knowledge that the results of this like any other surveys are based on respondent opinion rather than an on objective data.

One of the most striking outcomes of this survey, not investigated by previous ones, is the degree of variability in obtaining HD diagnosis. Although all respondents rely on a histologybased diagnosis and perform rectal biopsies as recommended,10 there is inconsistency in the way biopsies are obtained, processed, and interpreted. Almost two-thirds of respondents use the rectal suction technique, whereas more than a third opt for an open full-thickness biopsy. The difference is not just technical in specimen collection but has an implication in the requirement of sedation/anesthesia for the open approach. There is also a high degree of variability in the number of specimens collected and especially the location where the biopsy is taken. It is known that in the distal 1to $2 \mathrm{~cm}$ of the distal rectum the normal sparse nature of submucosal ganglion cells creates a challenge for the pathologist.11 These variations in practice show inconsisten $\neg$ cy and could have an effect on the different treatment modalities. The inconsistency in obtaining the specimens is also reflected on the pathology front, where there is a variability of markers used, with some being employed only in a minority of centers. Since the discovery that loss of calretinin immunostaining is a marker of ganglion cell absence in the aganglionic colon of HD patients,12 in many laboratories it has replaced acetylcholinesterase 
staining as it can be studied on formalin-fixed, paraffin-embedded sam ples.13 Some authors have even reported that calretinin immunostaining alone may be sufficient to exclude HD,14 although there are some scenarios where intact calretinin immunoreactive mucosal innervation is found in suction biopsies of HD patients ${ }^{13}$. According to our survey, calretinin immunostaining is not very popular as it is requested by only a third of the respondents.

In analyzing the results of the survey, we speculated that the volume of patients treated by a center could have an impact on the consistency of biopsy techniques, staining markers, and time to reach a diagnosis. However, we did not find any relationship between center volume and any of the parameters mentioned. For instance, among centers that manage more than 20 new cases of HD a year, 16\% reach a definitive diagnosis within 24 hours, $24 \%$ between 24 and 48 hours, 33\% between 3 and 5 days, and $27 \%$ in more than 5 days.

The degree of variability in obtaining and processing the rectal specimens might also have an impact on the different experience that certain centers have with intestinal dysgan $\neg$ glionoses, a heterogeneous group of anomalies of the enteric nervous system such as hypoganglionosis and intestinal neuronal dysplasia.15 Some surgeons have maintained a critical position regarding these controversial conditions as they consider these anomalies as secondary-acquired phe-nomena, sometimes associated with other diseases such as bowel atresia, HD, or simple constipation.

Contrast enema in patients with suspected HD is still very popular ( $96 \%$ respondents), although its utility either for HD diagnosis or for evaluation of the aganglionic segment length has been challenged.16,17 In a United Kingdom survey of practice only 38\% surgeons combine biopsies with a contrast enema.5 The Australian Pediatric Surveillance Unit recom $\neg$ mend that a contrast 
study should not be the final test to rule out HD, based on a study where only 56/126 (44\%) patients had preoperative contrast enema which was positive in 38 , giving an accuracy of 68\%.18 On the other hand, preoperative anorectal manometry is requested by only one-third of the respondents, maybe reflecting concerns regarding its accuracy in this age group. In the United Kingdom survey of practice, no surgeon re-ported the use of anorectal manometry in patients with suspected HD,5 whereas in a nationwide survey of 3,852 patients with HD managed over 30 years in Japan two-thirds of patients underwent manometry studies. The surgical management of HD remains one of the most controversial and diversified of all pediatric surgical condi-tions. Our survey shows that in a neonate with confirmed HD twothirds of respondents delay the PT for a period of time, and of these $27 \%$ create a stoma. This shows that a one-stage repair is performed by $71 \%$ of respondents, that is not too different from the proportion reported in the APSA survey (86\%).3 The majority of respondents who delay the PT plan the surgery when the infant is 3 months of age or more than $5 \mathrm{~kg}$. Various surgical techniques have been described and em-ployed over decades for the treatment of HD, but all stem from the approaches described by Orvar Swenson in 1948,19 Bernard Duhamel in 1956,20 or Franco Soave in 1963.21 The most popular surgical approach for the respondents of our survey is the transanal Soave PT, followed by the transanal Swenson. This is in line with the APSA survey, where the coloanal anastomosis was used by almost all responding surgeons, with only $5.4 \%$ preserving the aganglionic rectum with the Duhamel technique.3 Also in our survey, the Duha-mel is used by a smaller proportion of surgeons (16\%), whereas in the United Kingdom it is much more commonly used (38\%).5 Both the Japanese and the Australian surveys reported also that the Soave procedure is the commonest operation for definitive surgery. 8 
timing of the PT varies, but the majority of surgeons perform it between 1 and 6 months of age. This parallels the timing of the PT reported in other surveys.5,18 The management of patients with TCA is also variable. In the present survey, the majority of respondents (52\%) employ the Duhamel procedure for definitive surgery. This is in line with other surveys that report either the Duhamel or its modification such as the Lester-Martin.5,7 Some authors have proposed the J-pouch or S-pouch ileoanal anastomosis for patients with TCA after failed PT procedure.22,23 This ap-proach is chosen by one-third of the respondents of our survey.

We acknowledge the difficulty of interpreting some answers in this survey. As reported before, 9 in some coun-tries, surgical units are organized in a hierarchical model, whereby the chief of the unit holds most of the manage-ment decisions, whereas, in other countries, units are run by a group of consultants/attending surgeons, each of them representing an independent group with autonomy regard-ing clinical decision-making. Some of the questionnaires analyzed in the present survey may come from the same center. Therefore, if more than one surgeon from the same center opt for the same PT technique, it could be due to an independent choice or to a center-specific management choice. This is impossible to extrapolate given the anonym-ity of the response analysis.

In conclusion, the results of the present study highlight variation in the diagnosis and treatment of $\mathrm{HD}$ across the centers. This is reflected by the variability in obtaining rectal biopsies, the type of surgery, and the overall management of HD patients. The most common surgical technique used for PT is the transanal Soave for patients with standard segment HD. Opinions widely vary 
about the management of patients with TCA. Guidelines should be developed to avoid such degree of variability in management and to facilitate research studies. 


\section{References}

1 Grosfeld. Hirschsprung's disease: A historical perspective-1691- 2005. In: Holschneider A, Puri P, eds. Hirschsprung's Disease and Allied Disorders. Berlin, Germany: Springer-Verlag; 2008:1-12 Q8 Q8

2 Kleinhaus S, Boley SJ, Sheran M, Sieber WK. Hirschsprung's disease-a survey of the members of the Surgical Section of the American Academy of Pediatrics. J Pediatr Surg 1979;14(5): 588597

3 Keckler SJ, Yang JC, Fraser JD, et al. Contemporary practice patterns in the surgical management of Hirschsprung's disease. J Pediatr Surg 2009;44(6):1257-1260, discussion 1260 4 Huddart SN. Hirschsprung's disease: present UK practice. Ann R Coll Surg Engl 1998;80(1):4648

5 Bradnock TJ, Walker GM. Evolution in the management of Hirsch- sprung's disease in UK and Ireland: a national survey of practice revisited. Ann R Coll Surg Engl 2011;93(1):3438

6 Ikeda K, Goto S. Diagnosis and treatment of Hirschsprung's disease in Japan. An analysis of 1628 patients. Ann Surg 1984;199(4):400-405

7 Suita S, Taguchi T, Kamimura T, Yanai K. Total colonic aganglionosis with or without small bowel involvement: a changing profile. J Pediatr Surg 1997;32(11):1537-1541

8 Suita S, Taguchi T, leiri S, Nakatsuji T. Hirschsprung's disease in Japan: analysis of 3852 patients based on a nationwide survey in 30 years. J Pediatr Surg 2005;40(1):197-201, discussion 201-202

9 Zani A, Zani-Ruttenstock E, Eaton S, Pierro A. The value of surveys in pediatric surgery. Eur J Pediatr Surg 2015;25(6):500-503 
10 Noblett HR. A rectal suction biopsy tube for use in the diagnosis of Hirschsprung's disease. J Pediatr Surg $1969 ; 4(4): 406-409$ Aldridge RT, Campbell PE. Ganglion cell distribution in the normal rectum and anal canal. A basis for the diagnosis of Hirschsprung's disease by anorectal biopsy. J Pediatr Surg 1968;3(4):475-490

12 Barshack I, Fridman E, Goldberg I, Chowers Y, Kopolovic J. The loss of calretinin expression indicates aganglionosis in Hirschsprung's disease. J Clin Pathol 2004;57(7):712-716

13 Kapur RP, Reed RC, Finn LS, Patterson K, Johanson J, Rutledge JC. Calretinin immunohistochemistry versus acetylcholinesterase histochemistry in the evaluation of suction rectal biopsies for Hirschsprung Disease. Pediatr Dev Pathol 2009;12(1):6-15

14 Morris MI, Soglio DB, Ouimet A, Aspirot A, Patey N. A study of calretinin in Hirschsprung pathology, particularly in total colonic aganglionosis. J Pediatr Surg 2013;48(5):1037-1043

15 Martucciello G, Pini Prato A, Puri P, et al. Controversies concerning diagnostic guidelines for anomalies of the enteric nervous system: a report from the fourth International Symposium on Hirsch-sprung's disease and related neurocristopathies. J Pediatr Surg 2005;40(10):1527-1531 16 Chen JZ, Jamieson DH, Skarsgard ED. Does pre-biopsy contrast enema delay the diagnosis of long segment Hirschsprung's dis-ease? Eur J Pediatr Surg 2010;20(6):375-378

17 Muller CO, Mignot C, Belarbi N, Berrebi D, Bonnard A. Does the radiographic transition zone correlate with the level of aganglionosis on the specimen in Hirschsprung's disease? Pediatr Surg Int 2012;28(6):597-601

18 Singh SJ, Croaker GD, Manglick P, et al. Hirschsprung's disease: the Australian Paediatric Surveillance Unit's experience. Pediatr Surg Int 2003;19(4):247-250 
19 Swenson O, Bill AH Jr. Resection of rectum and rectosigmoid with preservation of the sphincter for benign spastic lesions producing megacolon; an experimental study. Surgery $1948 ; 24(2): 212-220$

20 Duhamel B. Retrorectal and transanal pull-through procedure for the treatment of Hirschsprung's disease. Dis Colon Rectum 1964; 7:455-458

21 Soave F. A new original technique for treatment of Hirschsprung's disease. Surgery $1964 ; 56: 1007-1014$

22 Rintala RJ, Lindahl HG. Proctocolectomy and J-pouch ileo-anal anastomosis in children. J Pediatr Surg 2002;37(1):66-70

23 Lal DR, Nichol PF, Harms BA, Go LL, Lund DP. Ileo-anal S-Pouch reconstruction in patients with total colonic aganglionosis after failed pull-through procedure. J Pediatr Surg 2004;39(7):e7-e9 


\section{Figure legends}

Fig. 1 Differences regarding rectal biopsies for the diagnosis of Hirschsprung disease: (A) Number of specimens routinely taken; (B) location of the most distal biopsy from the dentate line; (C) most used histological and immunohistochemical methods; (D) time to pathology report.

Fig. 2 Distribution of responses on the preferred definitive operation: (A) Type of pullthrough;(B)approach used for the pull-through.

Figure 1

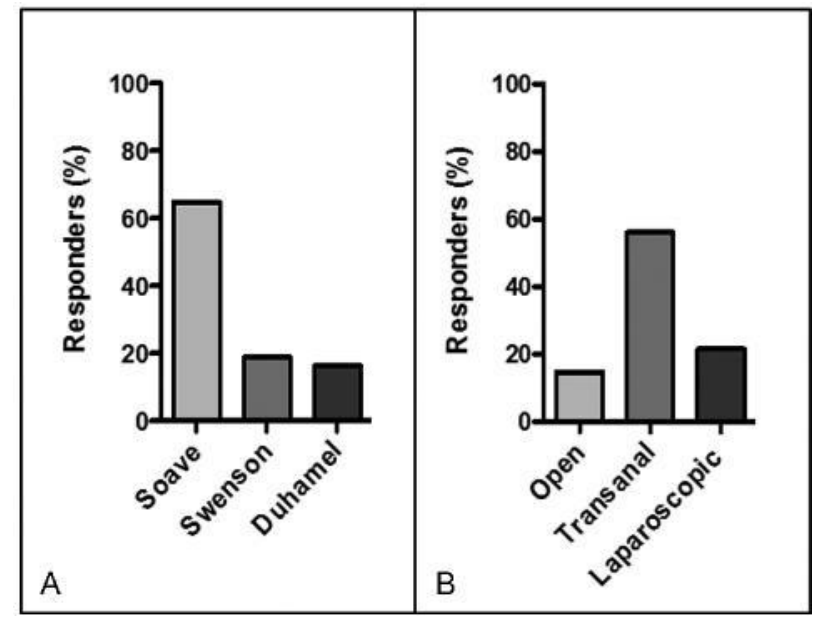

Figure 2 


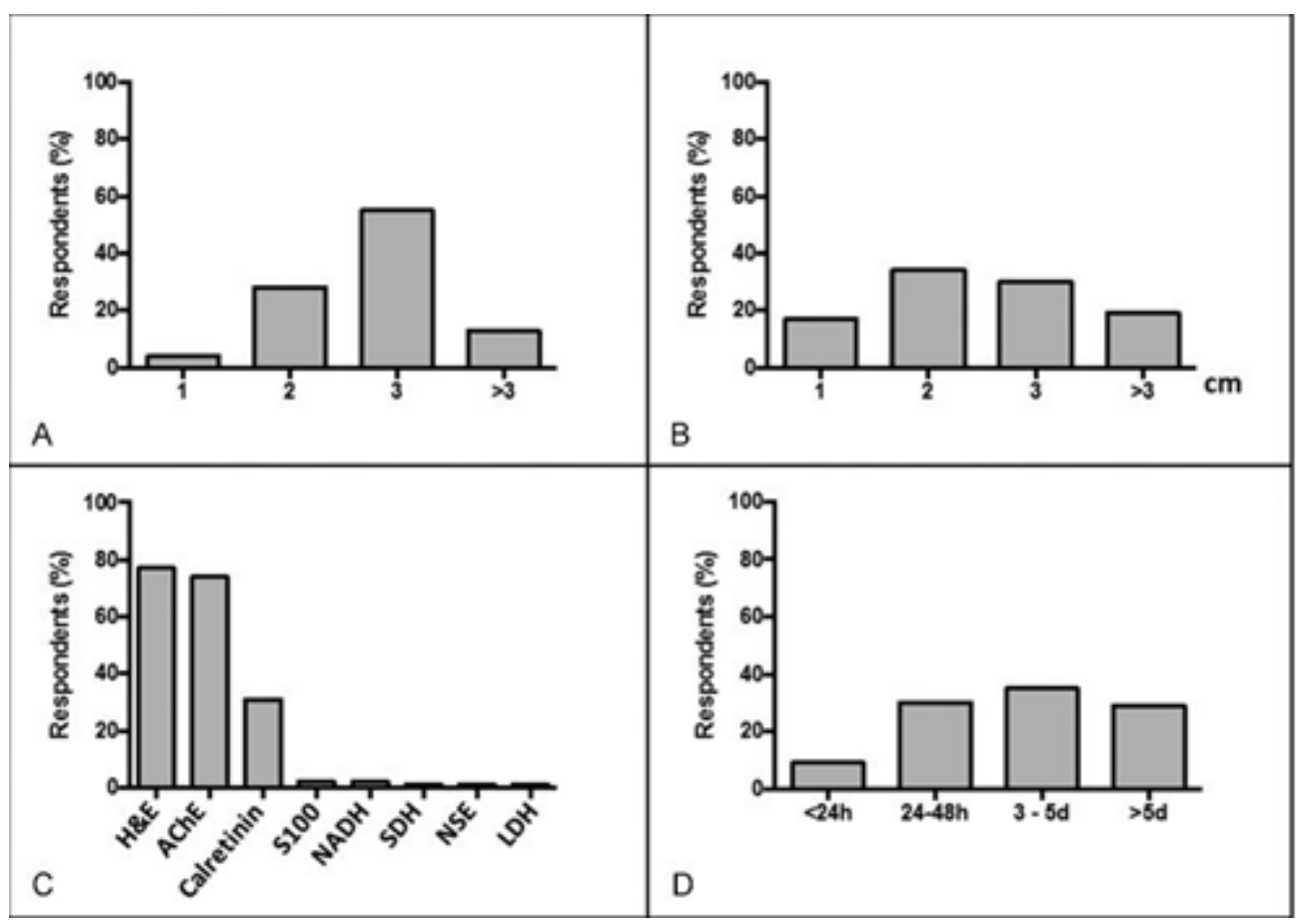

